\title{
A FIXED-POINT THEOREM FOR $p$-GROUP ACTIONS STEFAN JACKOWSKI
}

(Communicated by Haynes R. Miller)

\begin{abstract}
We prove Sullivan's fixed-point conjecture for fixed-point-free actions of compact Lie groups which are extensions of a $p$-group by a torus. Moreover, we show that for a finite $p$-group $G$ and a compact or finitely dimensional paracompact $G$-space $X$ the fixed point set $X^{G}$ is nonempty iff the induced homomorphism of zero-dimensional stable cohomotopy groups $\pi^{0}(B G) \rightarrow \pi^{0}\left(E G \times_{G} X\right)$ is injective.
\end{abstract}

Let $q^{G}: E G \rightarrow B G$ be a universal principal $G$-bundle. For a $G$-space $X$ consider the associated bundle $q_{X}^{G}: E G \times_{G} X \rightarrow B G$ with fiber $X$. Let $\pi^{0}(-)$ denote the zero-dimensional stable cohomotopy functor.

THEOREM A. Let $G$ be a finite p-group. Suppose that a $G$-space $X$ is compact, or is paracompact with finite cohomological dimension. Then the following conditions are equivalent:

(a) The fixed point set $X^{G}$ is nonempty,

(b) there exists a $G$-map $E G \rightarrow X$,

(c) $q_{X}^{G}$ has a section,

(d) the induced homomorphism $\left(q_{X}^{G}\right)^{*}: \pi^{0}(B G) \rightarrow \pi^{0}\left(E G \times_{G} X\right)$ is injective.

(For a definition of the cohomological dimension cf. Quillen [1971].)

Our theorem generalizes results of W. Y. Hsiang and T. tom Dieck. Hsiang $[1975$, IV.I] proved it on the assumption that $G$ is an elementary abelian $p$-group or a torus, and with singular cohomology replacing zero-dimensional stable cohomotopy in (d). T. tom Dieck [1972a] extended Hsiang's result to abelian compact Lie groups $G$ such that the group of components $G / G_{0}$ is a $p$-group, considering in (d) unitary cobordism theory instead of singular cohomology. In both theorems, in the case of a nondiscrete group $G$ and a noncompact space $X$, we have to assume additionally that $X$ has only finitely many orbit types. Proofs of those results are based on computations of appropriate cohomology of the classifying space of the acting group. Similary the proof of Theorem A relies on G. Carlsson's work (Carlsson [1984]) describing stable cohomotopy of classifying spaces.

Theorem A is related to a conjecture of D. Sullivan [1970, p. 5.118]. Suppose that $G$ and $X$ are as in Theorem A. The (generalized) Sullivan conjecture says that the map $X^{G} \rightarrow \operatorname{map}_{G}(E G, X)$ which assigns to every fixed point $x \in X^{G}$ the constant map $f_{x}: E G \rightarrow X$ induces an isomorphism of $\bmod p$ cohomology. For

Received by the editors February 6, 1986 and, in revised form, July 2, 1986.

1980 Mathematics Subject Classification (1985 Revision). Primary 55N25, 57S99.

Key words and phrases. p-group, fixed points, equivariant maps, sections, stable cohomotopy, the Sullivan conjecture, the Segal conjecture. 
the trivial $G$-action on $X$ it has been proved by H. Miller [1984]. The equivalence (a) $\Leftrightarrow$ (b) in Theorem A proves Sullivan's conjecture for fixed-point-free actions.

Theorems of Hsiang and tom Dieck suggest that Theorem A should generalize to a class of compact Lie groups. The following example shows that the largest class we can expect consists of extensions of finite $p$-groups by tori.

EXAMPLE 1. Let $G$ be a compact Lie group which is not an extension of a $p$-group by a torus. According to Edmonds and Lee [1976] there exists a smooth fixed-point-free $G$-action on a euclidean space $\mathbf{R}^{n}$. (Note that such action must have finitely many orbit types; see Bredon [1972, IV.I.3].) Clearly the bundle $E G \times{ }_{G} \mathbf{R}^{n} \rightarrow B G$ has a section; thus (a) and (c) are not equivalent.

The next example shows that condition (d) of Theorem A does not always imply existence of a fixed point, even for circle group actions.

EXAMPLE 2. Let $T$ be a circle group and let $X$ be a free $T$-orbit. Clearly $X^{T}=\varnothing$ but the homomorphism $\left(q_{x}^{T}\right)^{*}: \pi^{0}(B T) \rightarrow \pi^{0}\left(E T \times_{T} X\right)=\pi^{0}(\mathrm{pt})$ is known to be an isomorphism (Nishida [1983]).

However we have the following theorem, which we will deduce from Theorem A.

THEOREM B. Let $G$ be a compact Lie group such that its identity component $G_{0}$ is a torus group and $G / G_{0}$ is a p-group. Suppose that a $G$-space $X$ is either compact, or that $X$ is paracompact with finite cohomological dimensions, and has only a finite number of orbit types. Then conditions (a), (b), and (c) of Theorem A are equivalent.

ProOF OF THEOREM A. Implications (a) $\Rightarrow(\mathrm{b}) \Leftrightarrow(\mathrm{c}) \Rightarrow(\mathrm{d})$ are obvious. We prove $(\mathrm{d}) \Rightarrow(\mathrm{a})$. Consider the commutative diagram

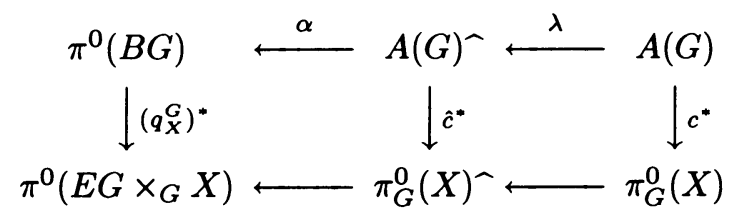

where $\pi_{G}^{0}(-)$ is the stable equivariant cohomotopy theory, $A(G)$ is the Burnside ring of $G$, and - denotes the completion with respect to the augmentation ideal $I(G)=\operatorname{ker}\{A(G) \rightarrow \mathbf{Z}\}$. Since $G$ is a $p$-group the completion map $\lambda$ restricted to the augmentation ideal $I(G)$ is just $p$-adic completion (Laitinen [1979]). The Burnside ring is a free abelian group hence $\lambda$ is monic. The Segal conjecture proved by Carlsson [1984] says that $\alpha$ is an isomorphism. Thus the above diagram and assumption (d) imply that the homomorphism $c^{*}$ induced by the constant map $c: X \rightarrow \mathrm{pt}$ is monic.

Assuming that $X^{G}=\varnothing$, we will produce a nonzero element $v \in A(G)$ such that $c^{*}(v)=0$. First we construct an element $u \in A(G)$ such that for every proper subgroup $H \varsubsetneqq G, u \in \operatorname{ker}\{$ res: $A(G) \rightarrow A(H)\}$. The element $u$ can be defined as a product of elements $u_{H}$ over all conjugacy classes $(H)$ of proper subgroups of $G$, where $u_{H}=[G / H]-\operatorname{card}\left((G / H)^{H}\right)[G / G]$. In order to see that $u \neq 0$, recall that elements of the Burnside ring are detected by the family of homomorphisms $\varphi_{H}: A(G) \rightarrow \mathbf{Z}$, where $H$ runs over all conjugacy classes of subgroups of $G$. For a $G$-set, $\varphi_{H}(S)=\operatorname{card}\left(S^{H}\right)$. Clearly, for any $H \varsubsetneqq G, \varphi_{H}(u)=0$ but $\varphi_{G}(u) \neq 0$. 
We have assumed that all isotropy subgroups on $X$ are proper. Hence the element $c^{*}(u)$ restricts trivially to every orbit which occurs on $X$. We will show that this implies that $c^{*}(u)$ is a nilpotent element in equivariant stable cohomotopy. Since $\pi_{G}^{*}(-)$ is an equivariant cohomology theory, it is enough to prove that every orbit $G x \subset X$ has a neighborhood $V_{x} \supset G x$ such that $c^{*}(u) \mid V_{x}=0$ (Quillen [1971, 3.2, 3.4]). The space $X$ is regular, thus each orbit $G x \subset X$ has an open $G$-invariant neighborhood $V_{x} \supset G x$ which admits an equivariant retraction $r_{x}: V_{x} \rightarrow G x$ onto the orbit (Bredon [1972, II.54.4]).

The diagram

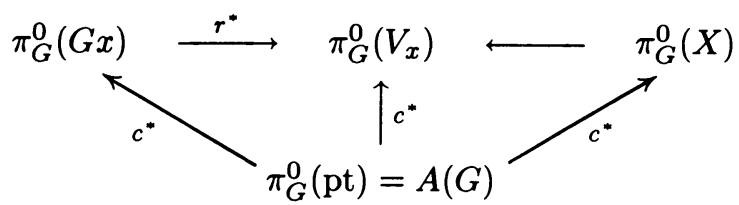

is clearly commutative, hence $c^{*}(u) \mid V_{x}=0$ and consequently $c^{*}\left(u^{n}\right)=c^{*}(u)^{n}=0$ for some $n>0$. The Burnside ring $A(G)$ does not contain nonzero nilpotent elements, so taking $v=u^{n}$ we reach a contradiction.

For the proof of Theorem B we need a lemma.

LEMMA. Let $G$ be a group such as in Theorem B. Suppose that $Y$ is a compact, fixed-point-free $G$-space. Then there exists a finite $p$-subgroup $P \subset G$ such that $Y^{P}=\varnothing$.

ProOF. Feshbach [1987] observed that there exists a nested sequence of finite $p$-subgroups of $G, P_{0} \subset P_{1} \subset \cdots$ which surject onto $G / G_{0}$ and whose union is dense in $G$. Clearly the fixed point sets of subgroups $P_{i}$ form a decreasing sequence of closed subsets $X^{P_{0}} \supset X^{P_{1}} \supset \cdots$ whose intersection is $X^{G}$. Since $X$ is compact, $X^{G}=\varnothing$ implies that $X^{P_{i}}=\varnothing$ for all sufficiently large $P_{i}$.

PROOF OF THEOREM B. Obviously a section of the bundle $q_{X}^{G}$ defines, for any subgroup $K \subset G$, a section of the bundle $q_{X}^{K}$. Thus, according to Theorem A, any finite $p$-subgroup $P \subset G$ has a fixed point. The last lemma implies the conclusion $X^{G} \neq \varnothing$ in the case of a compact space $X$.

Suppose now that $X$ is a finite-dimensional $G$-space with finitely many orbit types $\left(H_{1}\right), \ldots,\left(H_{n}\right)$, and that $X^{G}=\varnothing$. Consider the union of orbits $Y=G / H_{1} \cup$ $\cdots \cup G / H_{n}$. This is a compact $G$-space with empty fixed point set. Hence the lemma ensures existence of a finite $p$-subgroup $P \subset G$ such that $Y^{P}=\varnothing$. Because $\left(H_{i}\right)$, $i=1, \ldots, n$, were all orbit types which occur on $X$, we have also $X^{P}=\varnothing$. Since any finite $p$-subgroup of $G$ has a fixed point on $X$, we have reached a contradiction.

We conclude the paper by explaining a "relative" version of Theorem A. Suppose $G$ is an arbitrary finite group and $₹$ is a family of its subgroups. We say that a subgroup $K \subset G$ is a $p$-subgroup $\bmod \mathcal{F}$ if there exists a subgroup $H \in \mathcal{F}$ such that $H$ is normal in $K$ and $K / H$ is a $p$-group. Recall that a $G$-space $E \mp$ is called $\mathcal{F}$-universal if the fixed point set $(E \mathcal{F})^{H}$ is contractible whenever $H \in \mathcal{F}$, and is empty otherwise (cf. tom Dieck [1972b]).

THEOREM C. Let $\mathcal{F}$ be a family of subgroups of a finite group $G$. Suppose $X$ is a $G$-space which is compact, or is paracompact with finite cohomological dimen- 
sion. If the projection $q: X \times E \mathcal{F} \rightarrow E \mathcal{F}$ induces a monomorphism $q^{*}: \pi_{G}^{0}(E \mathcal{F}) \rightarrow$ $\pi_{G}^{0}(X \times E \mathcal{F})$, then $X^{K} \neq \varnothing$ whenever $K$ is a p-subgroup $\bmod \mathcal{F}$, for any prime number $p$.

Clearly, for a $p$-group $G$ and $₹$ consisting of the identity subgroup, Theorem $\mathrm{C}$ reduces to the implication (d) $\Rightarrow$ (a) of Theorem $A$. The proof of Theorem $C$, due to J.-P. Haeberly, is based on a computation of $\pi_{G}^{0}(E \mathcal{F})$ which generalizes the Segal conjecture (cf. Adams et al. [1984]). It will appear in his forthcoming paper.

ACKNOWLEDGEMENTS. I thank Haynes Miller and Jean-Pierre Haeberly for useful discussions. I am grateful to the referee for attentive reading of the manuscript and comments.

Added in April 1987. Since this paper was written, the implication (b) $\Rightarrow$ (a) of Theorem A was proved independently by E. Dror Farjoun and A. Zabrodsky. Later the Sullivan conjecture in its full generality was proved independently by G. Carlsson, H. Miller and J. Lannes. Their result clearly includes (a) $\Leftrightarrow$ (b) as its very special case. But the implication (d) $\Rightarrow$ (a) seems to be of a different character.

\section{BIBLIOGRAPHY}

[1986] J. F. Adams, J.-P. Haeberly, S. Jackowski, and J. P. May, A generalization of the Segal conjecture, Topology (to appear).

[1972] G. Bredon, Introduction to compact transformation groups, Pure Appl. Math., vol. 46, Academic Press, New York.

[1984] G. Carlsson, Equivariant stable cohomotopy and Segal's Burnside ring conjecture, Ann. of Math. 120, 189-224.

[1972a] T. tom Dieck, Existence of fixed points, Proc. Second Conf. on Compact Transformation Groups, Part I, Lecture Notes in Math., vol. 298, Springer-Verlag, Berlin and New York, pp. 163-169.

[1972b] _ Orbittypen und äquivariante Homologie. I, Arch. Math. 23, 307-317.

[1976] A. L. Edmonds and R. Lee, Compact Lie groups which act on euclidean space without fixed points, Proc. Amer. Math. Soc. 55, 416-418.

[1987] M. Feshbach, The Segal conjecture for compact Lie groups, Topology 26, 1-20.

[1975] W. Y. Hsiang, Cohomology theory of topological transformation groups, Ergeb. Math. Grenzgeb., Band 85, Springer-Verlag, Berlin and New York.

[1979] E. Laitinen, On the Burnside ring and stable cohomotopy of a finite group, Math. Scand. 44, 37-72.

[1984] H. Miller, The Sullivan conjecture on maps from classifying spaces, Ann. of Math. (2) 120, $39-87$.

[1983] G. Nishida, On the $S^{1}$-Segal conjecture, Publ. Res. Inst. Math. Sci. 19, 1153-1162.

[1971] D. Quillen, The spectrum of an equivariant cohomology ring. I, Ann. of Math. (2) 94, 549-572.

[1970] D. Sullivan, Geometric topology. Part I: Localization, periodicity and Galois symmetry, M.I.T. Press, Cambridge, Mass.

Instytut Matematyki, Uniwersytet WarszaWski, PKin IXP, Pl-00-901 WarSZAWA, POLAND 